UCRL-JC-127390

PREPRINT

\title{
Analysis of Classical Transport Equations for the Tokamak Edge Plasma
}

\author{
T.D. Rognlien \\ D.D. Ryutov
}

This paper was prepared for submittal to

Plasma Edge Theory Conference

Oxford, England

September 15-17, 1997

September 29, 1997

This is a preprint of a paper intended for publication in a joumal or proceedings. Since changes may be made before publication, this preprint is made available with the understanding that it will not be cited or reproduced without the permission of the author. 


\section{DISCLAIMER}

This document was prepared as an account of work sponsored by an agency of the United States Government. Neither the United States Government nor the University of California nor any of their employees, makes any warranty, express or implied, or assumes any legal liability or responsibility for the accuracy, completeness, or usefulness of any information, apparatus, product, or process disclosed, or represents that its use would not infringe privately owned rights. Reference herein to any specific commercial product, process, or service by trade name, trademark, manufacturer, or otherwise, does not necessarily constitute or imply its endorsement, recommendation, or favoring by the United States Government or the University of California. The views and opinions of authors expressed herein do not necessarily state or reflect those of the United States Government or the University of California, and shall not be used for advertising or product endorsement purposes. 


\title{
Analysis of Classical Transport Equations for the Tokamak Edge Plasma
}

\author{
T.D. Rognlien and D.D. Ryutov \\ Lawrence Livermore National Laboratory, Livermore, CA 94551, USA
}

\section{Introduction}

The classical fluid transport equations for a magneto-plasma as given, for example, by Braginskii [1], are complicated in their most general form. Here we obtain the simplest reduced set which contains the essential physics of the tokamak edge problem in slab geometry by systematically applying a parameter ordering and making use of specific symmetries. An important ingredient is a consistent set of boundary conditions as described elsewhere [2]. This model clearly resolves some important issues concerning diamagnetic drifts, high parallel viscosity, and the ambipolarity constraint. The final equations can also serve as a model for understanding the structure of the equations in the presence of anomalous transport terms arising from fluctuations. In fact, Braginskiilike equations are the basis of a number of scrape-off layer (SOL) transport codes [3]. However, all of these codes contain ad hoc radial diffusion terms and often neglect some classical terms, both of which make the self-consistency of the models questionable.

Braginskii's equations [1] have been derived from the first principles via the kinetic equations and, thereby, contain such "built-in" features as the symmetry of kinetic coefficients, and automatic quasineutrality of a cross-field diffusion in a system with toroidal symmetry such as a tokamak. Our model thus maintains these properties.

On the other hand, this set of equations is correct only within applicability limits that may be violated in the SOL using classical collision rates. For example, the thickness of the SOL, $\Delta_{x}$, in the case of low gas recycling, can be estimated as follows: The parallel life-time of a plasma element is $\tau_{\|} \sim L / v_{t i}$, where $L$ is the distance along the magnetic field, $\mathbf{B}$, from the midplane to the divertor or limiter, and $v_{t i}=\left(2 T_{i} / m_{i}\right)^{1 / 2}$. We assume that the ion and electron temperatures are of the same order $\left(T_{e} \sim T_{i}\right)$. The classical diffusion coefficient, $D_{c}$, across B is [1] $D_{c} \sim \rho_{e}^{2} \nu_{e} \sim \rho_{i}^{2} \nu_{i}\left(m_{e} / m_{i}\right)^{1 / 2}$ where $\rho_{i, e}=v_{t i, e} / \omega_{c e, i}, \omega_{c e, i}=e B / c m_{i, e}$, and $\nu_{i, e}$ and $m_{i, e}$ are the electron and ion collision frequencies and masses, respectively. Thus, in a parallel life-time, the radial diffusion broadens the SOL to the width

$$
\Delta_{x} \sim\left(D_{c} \tau_{\|}\right)^{1 / 2} \sim \rho_{i}\left(m_{e} / m_{i}\right)^{1 / 2}(L / \lambda)^{1 / 2}
$$

The classical fluid theory becomes invalid for $\Delta_{x}<\rho_{i}$, which can occur even for fairly strong collisionality when the mean-free path $\lambda \sim L\left(m_{e} / m_{i}\right)^{1 / 2} \sim L / 50$.

To avoid $\Delta_{x}<\rho_{i}$, and to make the analysis more directly applicable to present tokamak devices, we may enhance $\nu_{i, e}$ for the cross-field transport, but still maintain the ordering to be described. Such an enhancement has the advantage over ad hoc anomalous terms that the underlying conservations properties are still obeyed. Because the rapid parallel transport is believed in the range of classical values, the parallel 
terms need not be enhanced. Our focus is on the structure of the equations obtained and particularly on the calculation of the electrostatic potential.

\section{Geometry and Ordering}

We consider the slab geometry shown in Fig. 1a where all quantities are uniform in the $z$-direction. The $x$ and $y$ coordinates correspond to the poloidal and radial directions, respectively. The static magnetic field components, $B_{p} \equiv B_{x}$ and $B_{t} \equiv B_{z}$ lie in the $(y, z)$ plane. Material surfaces (limiter or divertor plates) exist at $y= \pm(d+p) / 2$, and periodic boundary conditions are applied at the edges of the core region given by $y= \pm d / 2$ for $x<0$. A private flux region exists for $x<0$ if $p>0$ to model divertors, whereas $p=0$ describes a limiter case. For $x>0$, B-field lines strike the plate, while for the core region, they close on themselves and the plasma requires periodic boundary conditions there.

It is convenient to use two coordinate systems in the $y-z$ plane related by a rotation angle of $\alpha=\arctan \left(B_{p} / B_{t}\right)$ shown in Fig. 1b. The $(y, z)$ coordinates have $z$ as an ignorable coordinate, and the $\left(y^{\prime}, z^{\prime}\right)$ coordinate system exploits the natural anisotropy of the transport coefficients with $z^{\prime}$ being along $B$. The various velocity components used are shown in Fig. $1 b$.

For our ordering, validity of the fluid analysis requires the two conditions [1]:

$$
\epsilon_{\lambda} \equiv \lambda / L \ll 1, \text { and } \epsilon_{\rho} \equiv \rho_{i} / \Delta_{x} \ll 1
$$

Additionally, we assume that the collision frequencies, $\nu_{i, e}=1 / \tau_{i, e}$, are much less than the corresponding gyrofrequencies, $\omega_{c i, c e}$, and that the SOL plasma is long and thin, i.e.,

$$
\epsilon_{\nu i, \nu e} \equiv \nu_{i, e} / \omega_{c i, c e} \ll 1, \text { and } \epsilon_{\Delta} \equiv \Delta_{x} /(d+p) \ll 1 .
$$

Also, the B-field makes a small angle with the plate: $\alpha \equiv \arctan \left(B_{P} / B_{T}\right) \ll 1$.

\section{Plasma Equations}

\subsection{General equations}

The basic aspects of our model can be obtained by considering the equations of continuity and momentum for electrons and ions [1]. We assume for now that the $T_{i, e}$ profiles are given; inclusion of the energy equations is discussed later.

The steady-state continuity equations have the form

$$
\nabla \cdot\left(n_{i, e} \mathbf{v}_{i, e}\right)=S_{i, e}^{p},
$$

where $n_{i, e}$ and $v_{i, e}$ are the electron and ion densities and velocities, respectively. The source term $S_{i, e}^{p}$ arises from ionization of neutral gas and recombination; generally $S_{e}^{p}=S_{i}^{p}$ for singly ionized plasmas unless a current source is present in the plasma.

The steady-state momentum equations are given by

$$
m_{i, e} n_{i, e} \mathbf{v}_{i, e} \cdot \nabla \mathbf{v}_{i, e}=-\nabla P_{i, e}+q n_{i, e}\left(\mathbf{E}+\mathbf{v}_{i, e} \times \mathbf{B} / c\right)-\mathbf{F}_{i, e}-\mathbf{R}_{i, e}+\mathbf{S}_{i, e}^{m} .
$$

Here $P_{i, e}=n_{i, e} T_{i, e}$ is the pressure, $q(= \pm e)$ is the particle charge, $\mathbf{E}$ is the electric field, $\mathbf{B}$ is the magnetic field, $c$ is the speed of light, $\mathbf{F}_{i, e}=\nabla \cdot \boldsymbol{\Pi}_{i, e}$ is the viscous force, and 
$\mathbf{R}_{i, e}$ is the thermal force [1]. The source $\mathbf{S}_{i, e}^{m}$ contains a sink term $-n m_{i, e} \mathbf{v}_{i} S_{i, e}^{p}$ which arises if newly created particles have no drift motion.

In their general three-dimensional form, the four equations given above represent eight partial differential equations for $n_{e}, n_{i}, v_{e}$, and $v_{i}$. The magnetic field is assumed static and given. The electrostatic potential, $\phi$, gives $\mathbf{E}=-\nabla \phi$. The equation for $\phi$ is from quasineutrality $\left(n_{e}=n_{i}\right)$; subtracting the two continuity equations gives the current continuity equation, $\nabla \cdot J=0$, where $J=n_{i} e\left(v_{i}-v_{e}\right)$. We shall reduce this set to three equations for the variables $n_{i}, v_{i \| l}$, and $\phi$.

\subsection{Dominant Force Terms and Velocities}

The subscript $i$ is dropped from ion mass, density, velocities, and forces while the less-used electron terms will always carry an $e$ subscript. The ion viscosity terms given by $F$ in Eq. (2) can be divided into $F_{0}, F_{1}$, and $F_{2}$ with terms proportional to $n T_{i} \tau_{i} \epsilon_{\nu i}^{\gamma}$ where $\gamma=0,1$ and 2, respectively [1]. The $F_{\| 0}$ term can be added as a correction to the pressure term in the form $\partial P_{i} / \partial y \rightarrow\left(\partial P_{i} / \partial y\right)\left[1+\mathcal{O}\left(\lambda_{i} / L\right)\right]$ where $\lambda_{i}$ is the ion mean-free path, and $L$ is the scale length of parallel variations. Thịs correction is thus small in the fluid regime and requires kinetic theory otherwise; we thus neglect it here.

The remaining $F$ terms for $\gamma=1,2$ are ( $F_{x}$ is small)

$$
F_{\| 1}+F_{\| 2}=\frac{\cos \alpha}{\omega_{c i}}\left[\frac{\partial P_{i}}{\partial y} \frac{\partial v_{\|}}{\partial x}-\frac{\partial P_{i}}{\partial x} \frac{\partial v_{\|}}{\partial y}\right]-4 \frac{\partial}{\partial x} \eta_{1} \frac{\partial v_{\|}}{\partial x}, \text { and } F_{\perp 2}=-\frac{\partial}{\partial x} \eta_{1} \frac{\partial v_{\perp}}{\partial x}
$$

where the viscosity coefficient is $\eta_{1}=(3 / 10)\left(m n T_{i} c / e B\right)\left(\omega_{c i} \tau_{i}\right)^{-1}$.

The $\mathbf{R}$ terms can be taken directly from Ref. [1] with appropriate modifications for our geometry, and noting that $\mathbf{R}_{i}=-\mathbf{R}_{\boldsymbol{e}}$. The dominant terms are $\left(R_{x}\right.$ is small)

$$
R_{\| e}=-c_{r e} m_{e} \nu_{e} \frac{J_{\|}}{e}+c_{t e} n \sin \alpha \frac{\partial T_{e}}{\partial y}, \text { and } R_{\perp e}=-\frac{1}{\omega_{c e} \tau_{e}}\left[\frac{\partial P_{t}}{\partial x}-\frac{3}{2} n \frac{\partial T_{e}}{\partial x}\right] \text {, }
$$

where the parallel current is $J_{\|}=n e\left(v_{\| i}-v_{\| e}\right), c_{r e} \approx 0.51$, and $c_{t e} \approx 0.71$.

To calculate $v_{\perp}$ and $v_{x}$ from Eq. (2), inertia may be neglected or included by iteration. Using the dominant terms of $\mathbf{F}$ and $\mathbf{R}$, one finds

$$
v_{\perp}=v_{D \perp}+v_{E \perp}-\frac{c S_{x}^{m}}{e n B}
$$

where $v_{D \perp}=(c / e n B)\left(\partial P_{i} / \partial x\right)$ and $v_{E \perp}=(c / B)(\partial \phi / \partial x)$ are the diamagnetic and $\mathbf{E} \times \mathbf{B}$ velocities, respectively. For $v_{x}$, the result is

$$
v_{x}=v_{D x}+v_{E x}+v_{F x}+v_{R x}+\frac{c S_{\perp}^{m}}{e n B}
$$

Here, $v_{D x}=-(c / e n B)\left(\cos \alpha \partial P_{i} / \partial y\right)$ and $v_{E x}=-(c / B)(\cos \alpha \partial \phi / \partial y)$. The velocity components arising from the viscosity is obtained using Eq. (4) for $F_{\perp}$, giving

$$
v_{F x}=\frac{1}{n} \frac{\partial}{\partial x} \frac{n \rho_{i}^{2} D_{i}}{T_{i}} \frac{\partial}{\partial x}\left(\frac{1}{n} \frac{\partial P_{i}}{\partial x}+e \frac{\partial \phi}{\partial x}\right) .
$$


where $D_{i} \equiv \eta_{1} / 0.3 n m=\left(T_{i} c / e B\right)\left(\omega_{c i} \tau_{i}\right)^{-1}$. We consider $\omega_{c i} \tau_{i}(\gg 1)$ a parameter that can be adjusted to give anomalous (Bohm-like) diffusion. For the momentum transfer term, we use Eq. (6) with $R_{\perp}=-R_{\perp \text { e }}$ to find

$$
v_{R x}=\frac{-D_{e}}{T_{e}}\left(\frac{1}{n} \frac{\partial P_{t}}{\partial x}-\frac{3}{2} \frac{\partial T_{e}}{\partial x}\right)
$$

where $D_{e} \equiv\left(T_{e} c / e B\right)\left(\omega_{c e} \tau_{e}\right)^{-1}$ is a another Bohm-like coefficient with $\omega_{c e} \tau_{e}$ being a second adjustable parameter.

For the electrons, viscosity and $S_{e}^{m}$ are negligible owing to $m_{e} \ll m_{i}$, giving

$$
v_{\perp e}=v_{D \perp e}+v_{E \perp}, \text { and } v_{x e}=v_{D x e}+v_{E x}+v_{R x} \text {, }
$$

where the electron diamagnetic velocity is $v_{D L_{e}}=-(c / e n B)\left(\partial P_{e} / \partial x\right)$, and $v_{D x e}=$ $(c / e n B)\left(\cos \alpha \partial P_{e} / \partial y\right)$.

\subsection{Final Equations for $v_{\|}, n$, and $\phi$}

Now that we have expressions for the perpendicular velocities, the final reduced differential equations for the variables $v_{\|}, n_{i}$, and $\phi$ can be obtained. The inertialess electron parallel momentum equation is used to eliminate $R_{\| i}\left(=-R_{\| e}\right)$ and $\partial \phi / \partial y$ in terms of $\partial P_{e} / \partial y$, giving

$$
m n\left[\left(v_{E_{x}}+v_{F_{x}}+v_{R x}\right) \frac{\partial v_{\|}}{\partial x}+\hat{v}_{y} \frac{\partial v_{\|}}{\partial y}\right]=-\sin \alpha \frac{\partial P_{t}}{\partial y}+4 \frac{\partial}{\partial x} \eta_{1} \frac{\partial v_{\|}}{\partial x}+S_{i}^{m},
$$

where $\hat{v}_{y} \equiv v_{\|} \sin \alpha+v_{E \perp} \cos \alpha$. Note that the gyroviscous term $F_{\|}$for $\gamma=1$ [first term in Eq. (3)] has cancelled with the diamagnetic terms on the left-hand side of Eq. (2).

The differential equation for $n$ comes from ion continuity, Eq. (1):

$$
\frac{\partial}{\partial x}\left[n\left(v_{E x}+v_{F x}+v_{R x}\right)\right]+\frac{\partial}{\partial y}\left(n \hat{v}_{y}\right)=S_{i}^{p} .
$$

Here the components of diamagnetic velocity, $\mathbf{v}_{D}$, have disappeared from the left-hand side because $\nabla \cdot n \mathbf{v}_{D}=0$.

The third differential equation is the current continuity equation, $\nabla \cdot \mathbf{J}=0$, obtained from subtracting the ion and electron continuity equations, to give

$$
\frac{\partial^{2}}{\partial x^{2}}\left[\frac{n \rho_{i}^{2} D_{i}}{T_{i}} \frac{\partial}{\partial x}\left(\frac{1}{n} \frac{\partial P_{i}}{\partial x}+e \frac{\partial \phi}{\partial x}\right)\right]+\sin \alpha \frac{\partial j_{\|}}{\partial y}=\left(\frac{c}{e B}\right)\left(-\frac{\partial S_{\perp}^{m}}{\partial x}+\cos \alpha \frac{\partial S_{x}^{m}}{\partial y}\right)
$$

Here we show explicitly how the momentum source terms can influence the potential equation. For example, using a ion-neutral collision frequency, $\nu_{n}$, gives $\mathbf{S}_{i}^{m}=$ $-\nu_{n} m_{i} n_{i} \mathbf{v}_{i}$, which can compete with $F_{\perp 2}$ in determining $j_{x}$, or there can be direct momentum input through neutral-beam sources.

Inclusion of the energy equation shows that the diamagnetic terms also cancel there, including the (5/2) $\left(c n_{i, e} T_{i, e} / q_{i, e} B^{2}\right) \mathbf{B} \times \nabla T_{i, e}$ heat flux. The radial heat diffusivity is $2 n D_{i}$ for the ions and $4.66 n_{e} D_{e}$ for the electrons [1]. The viscous heating makes a sub-dominant contributions to the energy equations. 


\section{Examples}

We illustrate the results of this model for parameters in the range of those observed in present-day tokamaks. The dimensions used (see Fig. 1a) are $a=2 \mathrm{~cm}, b=4 \mathrm{~cm}$, $d=1.5 \mathrm{~m}$, and $p=0.5 \mathrm{~m}$. The core midplane ion density is fixed to $5 \times 10^{19} \mathrm{~m}^{-3}$ with equal $T_{e}, i=100 \mathrm{eV}$. The parallel and poloidal velocities are zero on the core boundary. Divertor plate particle recycling is set to 0.9 for a diffusive neutral gas model. The B-field values are $B_{T}=3.5 \mathrm{~T}$ and $B_{P}=0.35 \mathrm{~T}$.

In Fig. 2, we show that $\phi$ is quite insensitive to the magnitude of $\eta_{1}$ for a case with $\omega_{c e} \tau_{e}=50$. Here, the changing value of $\omega_{c i} \tau_{i}$ only effects $\eta_{1}$ because the $T_{e, i}$ profiles are frozen in this case to values obtained for $\omega_{c i} \tau_{i}=50$ (see Fig. 4.). In the SOL, $J_{\|}$ dominants, and in the core, the poloidal integral of $J_{x}$ must vanish (with no sources) which does not depend on the magnitude of $\eta_{1}$. In Fig. 3, we show that the density profile is quite different when Eq. (10) is used for $v_{R x}$ compared to the density-diffusion model of $v_{x}=-D \partial n / \partial x$ for the same midplane value of $D=0.25 \mathrm{~m}^{2} / \mathrm{s}$. In Fig. 4, the energy equations are allowed to evolve using $D_{i, e}$ corresponding to $\omega_{c i, e} \tau_{i, e}=50$. The sensitivity of the full model to the diffusion coefficients via changes in $\omega_{c} \tau$ is shown in Fig. 5; at the larger value, poloidal $\mathbf{E} \times \mathbf{B}$ becomes large near the separatrix owing to the large $E_{x}$.

\section{Summary}

The results of a systematic analysis of the plasma fluid transport equations has be presented for the parameter ordering appropiate to the tokamak edge plasma. The effect of enhanced radial transport is modeled by increasing the classical values of two parameters, $\omega_{c i, e} \tau_{i, e}$, without the underlying conservation and symmetry properties of the system. The cancellation of diamagnetic terms and the role of viscosity for the potential are clearly illustrated.

Because the radial current, $J_{x}$, caused by the viscosity is small, other possible sources of $J_{x}$ should be considered [4]. For example, plasma flow in toroidal geometry give a centrifugal force that may drive such a current [4]. A upper bound on this current (there may be compensating terms) shows that for edge plasma paramters, it could be competitive with the viscous force. Also, a momentum source from collisions with neutrals could also compete, but for typical parameters, a neutral density in the range of $10^{18} \mathrm{~m}^{-3}$ is required.

\section{Acknowledgments}

We thank Drs. R. Cohen and N. Mattor for helpful discussions. Mattor has developed an anomalous viscosity coefficient that could replace $\eta_{1}$. This work was performed at LLNL under the auspices of the USDOE for contract number W-7405-ENG-48.

\section{References}

[1] Braginskii, S.I., Transport processes in a plasma, Reviews of Plasma Physics, Vol. I, Ed. M.A. Leontovich (Consultants Bureau, New York, 1965), p. 205.

[3] Cohen, R.H., and Ryutov, D.D., Phys. Plasmas 22011 (1995)

[3] Reiter, D., J. Nucl. Mat. 196-198 80 (1992).

[4] Chankin, A.V., J. Nucl. Mat. 241-243 199 (1997) 


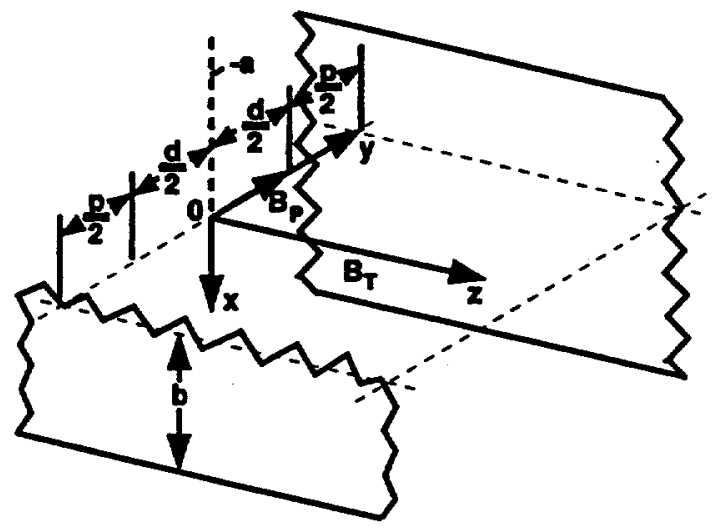

Fig. 1a. Coordinate system and regions used in the analysis showing static B-field components. Open field-lines exist $x>0$, whereas the core region with closed field-lines occupies the region $x<0$ and $-d / 2<y<d / 2$.

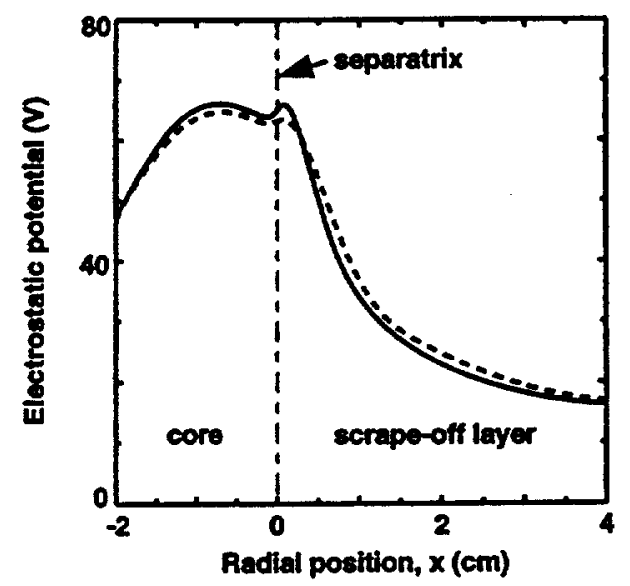

Fig. 2. Radial variation of potential at the midplane for two values of $\eta_{1}$ corresponding to $\omega_{c i} \tau_{i}=50$ (solid) $\omega_{\mathrm{ci}} \tau_{\mathrm{i}}=5$ (dashed). Temperature profiles fixed.

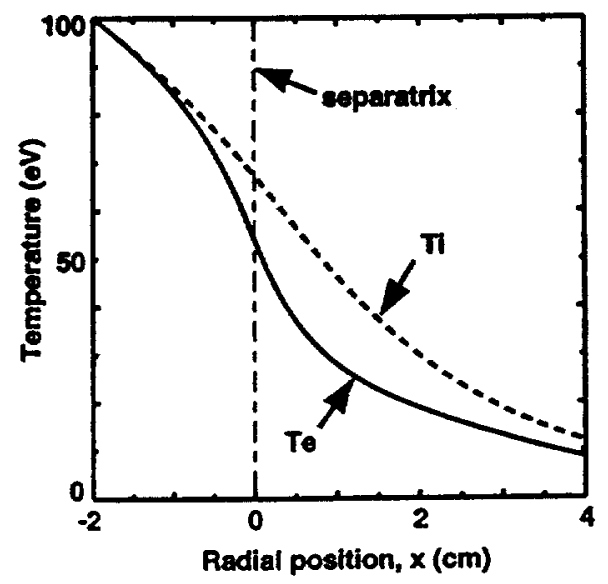

Fig. 4. Midplane temperature profiles for case where energy equations are evolved; $\omega_{c} \tau=50$ for ions and electrons.

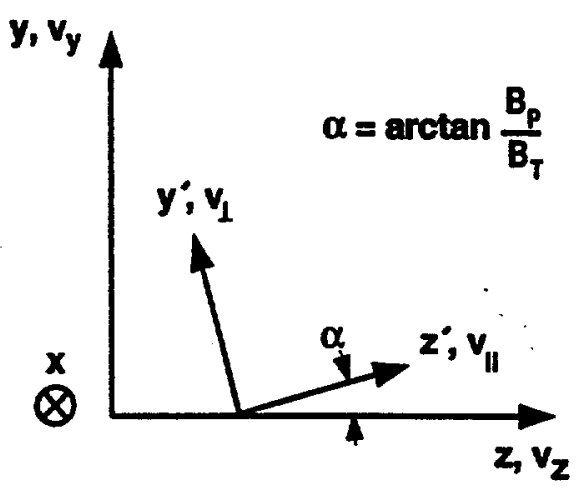

Fig. 1b. The $(y, z)$ plane showing the second coordinate system $\left(y^{\prime}, z^{\prime}\right)$ and velocities used to separate parallel and cross-field terms.

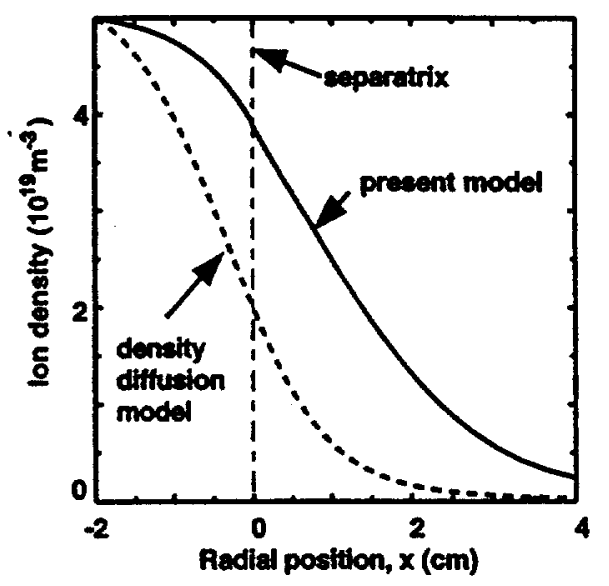

Fig. 3. Density profiles for case in Fig. 2 using present model diffusion [Eq. (10)] compared to simple density-gradient velocity with same $D$.

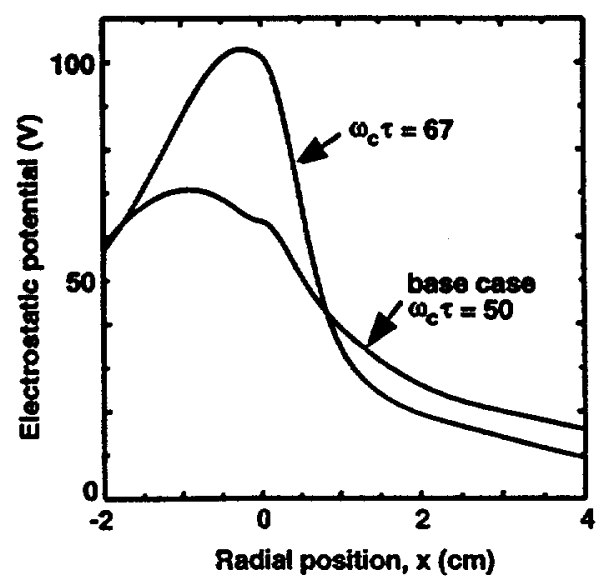

Fig. 5. Midplane potential for two different effective diffusion coefficients with the energy equations evolved. 


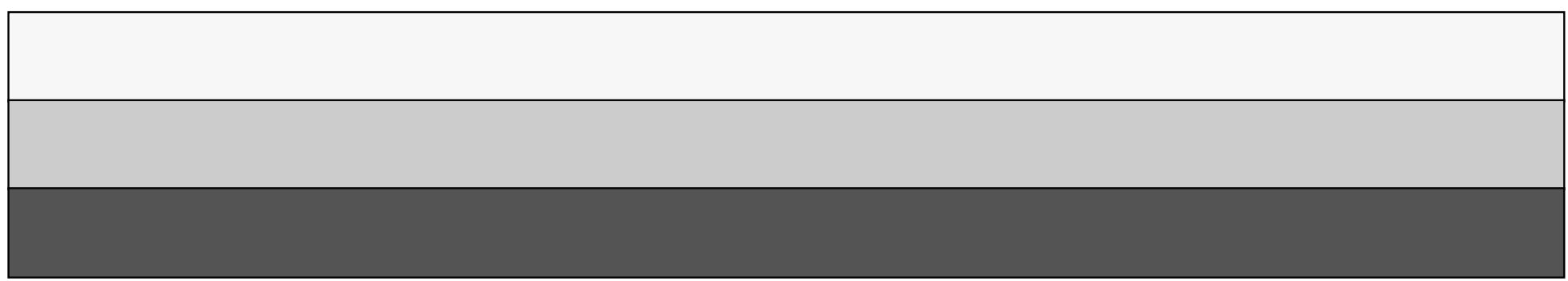

